\title{
Newton-type regularization methods for nonlinear inverse problems
}

\author{
$\underline{\text { Qinian Jin }}^{\text {a }}$ \\ ${ }^{a}$ Mathematical Sciences Institute, The Australian National University, Canberra, ACT 0200, Australia \\ Email: Qinian.Jin@anu.edu.au
}

\begin{abstract}
Inverse problems arise whenever one searches for unknown causes based on observation of their effects. Such problems are usually ill-posed in the sense that their solutions do not depend continuously on the data. In practical applications, one never has the exact data; instead only noisy data are available due to errors in the measurements. Thus, the development of stable methods for solving inverse problems is an important topic.

In the last two decades, many methods have been developed for solving nonlinear inverse problems. Due to their straightforward implementation and fast convergence property, more and more attention has been paid on Newton-type regularization methods including the general iteratively regularized Gaus-newton methods and the inexact Newton regularization methods.

The iteratively regularized Gauss-Newton method was proposed by Bakushinski for solving nonlinear inverse problems in Hilbert spaces, and the method was quickly generalized to its general form. These methods produce all the iterates in some trust regions centered around the initial guess. The regularization property was explored under either a priori or a posteriori stopping rules. We will present our recent convergence results when the discrepancy principle is used to terminate the iteration.

The inexact Newton regularization methods was initiated by Hanke and then generalized by Rieder to solve nonlinear inverse problems in Hilbert spaces. In contrast to the iteratively regularized GaussNewton methods, such methods produce the next iterate in a trust region centered around the current iterate by regularizing local linearized equations. An approximate solution is output by a discrepancy principle. Although numerical simulation indicates that they are quite efficient, for a long time it has been an open problem whether the inexact Newton methods are order optimal. We will report our recent work and confirm that the methods indeed are order optimal.
\end{abstract}

In some situations, regularization methods formulated in Hilbert space setting may not produce good results since they tend to smooth the solutions and thus destroy the special feature in the exact solution. On the other hand, many inverse problems can be more naturally formulated in Banach spaces than in Hilbert spaces. Therefore, it is necessary to develop regularization methods in the framework of Banach spaces. By making use of duality mappings and Bregman distance we will indicate how to formulate some Newton-type methods in Banach space setting and present the corresponding convergence results.

Keywords: Nonlinear inverse problems, the iteratively regularized Gauss-Newton methods, the inexact Newton methods, discrepancy principle, convergence, order optimality 


\section{INTRODUCTION}

Nonlinear inverse problems arise from many practical applications that include parameter identifications in partial differential equations, inverse scattering problems, tomographies, and biomedical imaging and so on. Mathematically, a nonlinear inverse problem usually can be formulated as the problem of finding a solution $x^{\dagger}$ of the operator equation

$$
F(x)=y,
$$

where $F: D(F) \subset X \mapsto Y$ is a Fréchet differentiable nonlinear operator between two Banach spaces $X$ and $Y$ with domain $D(F)$.

Inverse problems are usually ill-posed in the sense that their solutions do not depend continuously on the data. In practical applications, one never has exact data, but only noisy data are available due to errors in the measurements. Let $y^{\delta}$ be some noisy data satisfying $\left\|y^{\delta}-y\right\| \leq \delta$ with a given small noise level $\delta>0$. In order to obtain a stable approximation to $x^{\dagger}$ from $y^{\delta}$, one has to use regularization methods.

Many regularization methods have been developed for solving nonlinear inverse problems. One of the well-known methods is Tikhonov regularization which defines the regularized solution as the minimizer of the minimization problem

$$
\min _{x \in D(F)}\left\{\left\|F(x)-y^{\delta}\right\|^{2}+\alpha\left\|x-x_{0}\right\|^{2}\right\}
$$

The convergence properties as well as a posteriori rules for choosing the regularization parameter $\alpha$ have been considered extensively, see Engl, Kunisch and Neubauer (1989), Scherzer, Engl and Kunisch (1993), Jin and Hou (1999) and Tautenhahn and Jin (2003). Since the functional in (2) is in general non-convex, additional effort is required to find a minimizer which makes Tikhonov regularization rather expensive.

Due to their straightforward implementation and fast convergence property, Newton type methods becomes more and more popular for solving nonlinear inverse problems. In this survey, we will focus on two types of Newton methods: the general iteratively regularized Gauss-Newton methods and the inexact Newton methods. We will give the detail description on these methods and present recent relevant results on their convergence properties.

\section{NEWTON-TYPE METHODS IN HILBERT SPACES}

In this section we assume that both $X$ and $Y$ are Hilbert spaces and use $F^{\prime}(x)$ to denote the Fréchet derivative of $F$ at $x \in D(F)$. In order to describe the Newton type methods, we start with an initial guess $x_{0} \in D(F)$. Assume that $x_{n}$ is the current iterate, we replace $F(x)$ by its linearization around $x_{n}$ and obtain from (1) the the approximate equation

$$
F^{\prime}\left(x_{n}\right)\left(x-x_{n}\right)=y^{\delta}-F\left(x_{n}\right) .
$$

If $F^{\prime}\left(x_{n}\right)$ has bounded inverse, the usual Newton method defines the next iterate by solving (3) for $x$. For nonlinear inverse problems, however, $F^{\prime}\left(x_{n}\right)$ in general is not invertible. One should apply regularization methods to (3) or its variant to produce the next iterate.

\subsection{The general iteratively regularized Gauss-Newton methods}

In order to formulate this type of Newton methods, we rewrite (3) as

$$
F^{\prime}\left(x_{n}\right)\left(x-x_{0}\right)=y^{\delta}-F\left(x_{n}\right)+F^{\prime}\left(x_{n}\right)\left(x_{n}-x_{0}\right) .
$$

We take a sequence of positive numbers $\left\{\alpha_{n}\right\}$ satisfying

$$
\alpha_{n}>0, \quad 1 \leq \frac{\alpha_{n}}{\alpha_{n+1}} \leq r \quad \text { and } \quad \lim _{n \rightarrow \infty} \alpha_{n}=0
$$

for some constant $r>1$. Letting $\left\{g_{\alpha}\right\}$ be a family of spectral filter functions and applying the corresponding linear regularization method defined by $\left\{g_{\alpha}\right\}$ to (4), it leads to the general iteratively regularized Gauss-Newton methods

$$
\left.x_{n+1}=x_{0}-g_{\alpha_{n}}\left(T_{n}^{*} T_{n}\right)\right) T_{n}^{*}\left(F\left(x_{n}\right)-y^{\delta}-T_{n}\left(x_{n}-x_{0}\right)\right),
$$


where $T_{n}:=F^{\prime}\left(x_{n}\right)$ and $T_{n}^{*}$ denotes the adjoint of $T_{n}$. The function $\left\{g_{\alpha}\right\}$ in (6) can be chosen in various ways to produce various iterative methods. For the function $g_{\alpha}(\lambda)=1 /(\alpha+\lambda)$ arising from the Tikhonov regularization, (6) becomes

$$
x_{n+1}=x_{n}-\left(\alpha_{n} I+T_{n}^{*} T_{n}\right)^{-1}\left(T_{n}^{*}\left(F\left(x_{n}\right)-y^{\delta}\right)+\alpha_{n}\left(x_{n}-x_{0}\right)\right),
$$

which is the iteratively regularized Gauss-Newton method of Bakushinskii (1992). For the function $g_{\alpha}(\lambda)=\left[1-(1-\lambda)^{[1 / \alpha]}\right] / \lambda$ arising from the linear Landweber iteration, where $[1 / \alpha]$ denotes the largest integer not greater than $1 / \alpha$, the method (6) becomes

$$
\begin{aligned}
u_{n, 0} & :=x_{0}, \\
u_{n, j+1} & :=u_{n, j}-T_{n}^{*}\left(F\left(x_{n}\right)-y^{\delta}-T_{n}\left(x_{n}-u_{n, j}\right)\right), \quad 0 \leq j \leq\left[1 / \alpha_{n}\right]-1, \\
x_{n+1} & :=u_{n,\left[1 / \alpha_{n}\right]},
\end{aligned}
$$

For other different choice of $\left\{g_{\alpha}\right\}$, one may consult Kaltenbacher (1997) and Jin and Tautenhahn (2009).

When used to solve ill-posed inverse problems, iterative methods in general show the semi-convergence property. Therefore, the iteration must be terminated properly. Considering the practical applications, it is necessary to use the a posteriori information, i.e. all available data during computation, to choose the stopping index of iteration yielding order optimal convergence rates. The discrepancy principle

$$
\left\|F\left(x_{n_{\delta}}\right)-y^{\delta}\right\| \leq \tau \delta<\left\|F\left(x_{n}\right)-y^{\delta}\right\|, \quad 0 \leq n<n_{\delta},
$$

where $\tau>1$ is a given number, is a well-known stoping rule and has been applied successfully to regularization methods for linear ill-posed problems. This principle outputs an integer $n_{\delta}$ and hence an approximate solution $x_{n_{\delta}}$. By establishing the key inequality

$$
\left\|x_{n_{\delta}}-x^{\dagger}\right\| \lesssim\left\|x_{n}-x^{\dagger}\right\|+\frac{1}{\sqrt{\alpha_{n}}}\left(\left\|F\left(x_{n_{\delta}}\right)-y^{\delta}\right\|+\delta\right), \quad n \geq n_{\delta}
$$

connecting the error terms with the discrepancy, the convergence behavior of $x_{n_{\delta}}$ to $x^{\dagger}$ has been analyzed carefully in Jin and Tautenhahn (2009) where the main results can be summarized roughly as follows.

Theorem 1. Let $\tau>1$ and let $\left\{\alpha_{n}\right\}$ satisfies (5). If $F^{\prime}$ satisfies the Lipschitz condition

$$
\left\|F^{\prime}(x)-F^{\prime}(z)\right\| \leq L\|x-z\|, \quad \forall x, z \in B_{\rho}\left(x^{\dagger}\right) \subset D(F),
$$

then, for a large class of spectral filter functions $g_{\alpha}$, the method (6) and the discrepancy principle (7) define an order optimal regularization method for each $1 / 2 \leq \nu \leq \bar{\nu}-1 / 2$, i.e.

$$
\left\|x_{n_{\delta}}-x^{\dagger}\right\| \leq C_{\nu}\|\omega\|^{1 /(1+2 \nu)} \delta^{2 \nu /(1+2 \nu)}
$$

if $x_{0}-x^{\dagger}$ satisfies the source condition $x_{0}-x^{\dagger}=\left(F^{\prime}\left(x^{\dagger}\right)^{*} F^{\prime}\left(x^{\dagger}\right)\right)^{\nu} \omega$ for some $\omega \in X$ and $1 / 2 \leq \nu \leq$ $\bar{\nu}-1 / 2$. where $\bar{\nu} \geq 1$ denotes the qualification of $\left\{g_{\alpha}\right\}$.

Moreover, if $F$ satisfies the stronger condition that there exist constants $K_{0}$ and $K_{1}$ such that

$$
\left\|\left[F^{\prime}(x)-F^{\prime}(z)\right] w\right\| \leq K_{0}\|x-z\|\left\|F^{\prime}(z) w\right\|+K_{1}\left\|F^{\prime}(z)(x-z)\right\|\|w\|
$$

for $x, z \in B_{\rho}\left(x^{\dagger}\right) \subset D(F)$ and $w \in X$, then the method is also order optimal for $0<\nu \leq 1 / 2$ and $x_{n_{\delta}} \rightarrow x^{\dagger}$ as $\delta \rightarrow 0$ without any source condition.

The discrepancy principle (7) has been also considered in Blaschke, Neubauer and Scherzer (1997) for the iteratively regularized Gauss-Newton method of Bakushinskii; the number $\tau$ in (7), however, was required to be sufficiently large. It is worthy to mention that Theorem 1 requires only $\tau>1$ which is significant in practical applications.

There are other stopping rules proposed for the general iteratively regularized Gauss-Newton methods; for instance, a variant of the discrepancy principle has been considered in Kaltenbacher (1998), a variant of the rule in Scherzer, Engl and Kunisch (1993) has been adapted in Jin (2000), and a Lepskij-type stopping rule has been proposed in Bauer and Hohage (2005). 


\subsection{Inexact Newton methods}

Motivated by the inexact Newton methods in Demo, Eisenstat and Steihaug (1982) for well-posed problems, Hanke proposed his regularzing Levenberg-Marquardt scheme in Hanke (1997) for solving nonlinear inverse problems. The idea was then generalized in Rieder (1999) to introduce a general class of inexact Newton regularization methods, see also Lechleiter and Rieder (2010).

Every inexact Newton method consists of two components: an outer Newton iteration and an inner scheme providing increments by regularizing the local linearized equation. An approximate solution is output by a discrepancy principle. To be more precise, the method starts with an initial guess $x_{0} \in D(F)$. Assume that $x_{n}$ is a current iterate, one may apply any regularization scheme to the linearized equation (3) to produce a family of regularized approximations $\left\{x_{n}(t)\right\}$. One may choose $t_{n}$ to be the smallest number $t_{n}>0$ such that

$$
\left\|y^{\delta}-F\left(x_{n}\right)-F^{\prime}\left(x_{n}\right)\left(x_{n}\left(t_{n}\right)-x_{n}\right)\right\| \leq \mu\left\|y^{\delta}-F\left(x_{n}\right)\right\|
$$

for some preassigned value $0<\mu<1$. The next iterate is then updated as $x_{n+1}:=x_{n}\left(t_{n}\right)$. The outer Newton iteration is terminated by the discrepancy principle (7) for some given number $\tau>1$. This outputs an integer $n_{\delta}$ and hence $x_{n_{\delta}}$ which is used to approximate the exact solution $x^{\dagger}$.

The convergence rates of inexact Newton regularization methods have been considered in Rieder (1999) and Rieder (2001). However, only suboptimal result has been derived. It is a long-standing question whether the inexact Newton regularization methods are order optimal. Important progress has been made recently in Hanke (2010) where the regularizing Levenberg-Marquardt scheme is proved to be order optimal. In Jin (2011b) we considered the inexact Newton regularization methods in which the inner scheme defines

$$
x_{n}(t)=x_{n}+g_{t}\left(F^{\prime}\left(x_{n}\right)^{*} F^{\prime}\left(x_{n}\right)\right) F^{\prime}\left(x_{n}\right)^{*}\left(y^{\delta}-F\left(x_{n}\right)\right)
$$

with the spectral filter functions

$$
g_{t}(\lambda)=\sum_{j=0}^{[t]-1}(1-\lambda)^{j}, \quad \sum_{j=1}^{[t]}(1+\lambda)^{-j}, \quad \frac{1}{\lambda}\left(1-e^{-t \lambda}\right), \quad\left(\frac{1}{t}+\lambda\right)^{-1}
$$

arising from Landweber iteration, the implicit iteration, the asymptotic regularization, and Tikhonov regularization respectively, and obtained the order optimality which is contained in the following result.

Theorem 2. Assume that $F$ is properly scaled so that $\left\|F^{\prime}(x)\right\| \leq \Theta<1$ for all $x \in B_{\rho}\left(x^{\dagger}\right) \subset D(F)$ and satisfies the Newton-Mysovskii condition, i.e. there exists $K_{0} \geq 0$ such that

$$
\left\|\left[F^{\prime}(x)-F^{\prime}(z)\right] h\right\| \leq K_{0}\|x-z\|\left\|F^{\prime}(z) h\right\|, \quad \forall h \in X \text { and } x, z \in B_{\rho}\left(x^{\dagger}\right) .
$$

Let $\tau>2$ and $0<\mu<1$ be such that $\tau \mu>2$, and let $x_{0} \in B_{\rho}\left(x^{\dagger}\right)$. If $K_{0}\left\|x_{0}-x^{\dagger}\right\|$ is small, then the above inexact Newton regularization methods are well-defined and terminate after $n_{\delta}=O(1+|\log \delta|)$ iterations. If, in addition, $x_{0}-x^{\dagger}=\left(F^{\prime}\left(x^{\dagger}\right)^{*} F^{\prime}\left(x^{\dagger}\right)\right)^{\nu} \omega$ for some $\omega \in \mathcal{N}\left(F^{\prime}\left(x^{\dagger}\right)\right)^{\perp} \subset X$ and $0<\nu \leq$ $1 / 2$, then there holds

$$
\left\|x_{n_{\delta}}-x^{\dagger}\right\| \leq C\|\omega\|^{1 /(1+2 \nu)} \delta^{2 \nu /(1+2 \nu)}
$$

for some constant $C$ independent of $\delta$ and $\|\omega\|$.

This theorem extends the order optimality result in Hanke (2010) to a general class of inexact Newton regularization methods. These methods have been considered also in Hilbert scales in Jin (2011b). As far as the convergence of these methods without source conditions be concerned, it has been proved to be true for the exact data case in Lechleiter and Rieder (2010); however, it remains open for the noisy data case.

One may consider the above methods with $\left\{t_{n}\right\}$ given a priori. This has the advantage to save the effort to compute $t_{n}$ in some situations. To be more general, we formulate such methods in Hilbert scales which 
consists of a family of Hilbert spaces $\left\{X_{r}\right\}_{r \in \mathbb{R}}$ induced by a densely defined self-adjoint strictly positive linear operator $L$ in $X$, where each $X_{r}$ is the completion of $\cap_{k=0}^{\infty} D\left(L^{k}\right)$ with respect to the Hilbert space norm $\|x\|_{r}:=\left\|L^{r} x\right\|_{X}$. These methods takes the form

$$
x_{n+1}=x_{n}-g_{t_{n}}\left(L^{-2 s} F^{\prime}\left(x_{n}\right)^{*} F^{\prime}\left(x_{n}\right)\right) L^{-2 s} F^{\prime}\left(x_{n}\right)^{*}\left(F\left(x_{n}\right)-y^{\delta}\right),
$$

where $s \in \mathbb{R}$ is a preassigned number and $\left\{t_{n}\right\}$ is a sequence of positive numbers satisfying

$$
s_{n+1} \leq c_{0} s_{n} \quad \text { and } \quad t_{n} \geq c_{1}, \quad n=0,1, \cdots
$$

for some $c_{0}>1$ and $c_{1}>0$, where $s_{n}=\sum_{j=0}^{n} t_{j}$. The spectral filter functions $\left\{g_{t}\right\}$ can be chosen in various ways. If we choose $g_{t}(\lambda)=\sum_{j=0}^{[t]-1}(1-\lambda)^{j}$ with $t_{n}=1$ for all $n$, then (12) with $s=0$ becomes the nonlinear Landweber iteration (see Hanke, Neubauer and Scherzer (1995))

$$
x_{n+1}=x_{n}-F^{\prime}\left(x_{n}\right)^{*}\left(F\left(x_{n}\right)-y^{\delta}\right),
$$

which is known to be a slow convergent method. The flexibility in choosing $\left\{t_{n}\right\}$ can reduce significantly the computational work by computing more cheap steps in each inner iteration.

The following order optimality result has been proved in Jin and Tautenhahn (2011) when the method (12) is terminated by the discrepancy principle (7).

Theorem 3. Let $F$ satisfy the following conditions:

(a) There exists $a \geq 0$ and $0<m \leq M<\infty$ such that $m\|h\|_{-a} \leq\left\|F^{\prime}(x) h\right\| \leq M\|h\|_{-a}$ for all $h \in X$ and $x \in B_{\rho}\left(x^{\dagger}\right)$;

(b) $\left\|F^{\prime}(x) L^{-s}\right\|_{X \rightarrow Y} \leq 1$ for all $x \in B_{\rho}\left(x^{\dagger}\right)$, where $s \geq-a$;

(c) There exist $0<\beta \leq 1,0 \leq b \leq a$ and $K_{0} \geq 0$ such that

$$
\left.\| F^{\prime}(x)^{*}-F^{\prime} x^{\dagger}\right)^{*}\left\|_{Y \rightarrow X_{b}} \leq K_{0}\right\| x-x^{\dagger} \|^{\beta}, \quad x \in B_{\rho}\left(x^{\dagger}\right) .
$$

Let $\tau>1$ and let $\left\{t_{n}\right\}$ satisfy (13). If $x_{0}-x^{\dagger} \in X_{\mu}$ for some $(a-b) / \beta<\mu \leq b+2 s$, then, for the functions $\left\{g_{t}\right\}$ given by (11), the methods (12) coupled with the discrepancy principle (7) are well-defined, and for all $r \in[-a, \mu]$ there holds

$$
\left\|x_{n_{\delta}}-x^{\dagger}\right\|_{r} \leq C\left\|x_{0}-x^{\dagger}\right\|_{\mu}^{(a+r) /(a+\mu)} \delta^{(\mu-r) /(a+\mu)} .
$$

\section{NEWTON-TYPE METHODS IN BANACH SPACES}

In this section we consider equation (1) with $F$ being a nonlinear operator between two Banach spaces $X$ and $Y$. We use $X^{*}$ to denote the dual space of $X$ and use $\left\langle x^{*}, x\right\rangle$ to denote the dual pair for $x \in X$ and $x^{*} \in X^{*}$. We will assume that $X$ is uniformly covex and uniformly smooth, where $X$ is called uniformly convex if its modulus of convexity

$$
\delta_{X}(\epsilon):=\inf \{2-\|x+\bar{x}\|:\|x\|=\|\bar{x}\|=1,\|x-\bar{x}\| \geq \epsilon\}
$$

satisfies $\delta_{X}(\epsilon)>0$ for all $0<\epsilon \leq 2$, while $X$ is called uniformly smooth if its modulus of smoothness

$$
\rho_{X}(s):=\sup \{\|x+\bar{x}\|+\|x-\bar{x}\|-2:\|x\|=1,\|\bar{x}\| \leq s\}
$$

satisfies $\lim _{s \rightarrow 0} \frac{\rho_{X}(s)}{s}=0$. For each $1<p<\infty$ the set-valued mapping $J_{p}: X \rightarrow X^{*}$ defined by

$$
J_{p}(x):=\left\{x^{*} \in X^{*}:\left\|x^{*}\right\|=\|x\|^{p-1} \text { and }\left\langle x^{*}, x\right\rangle=\|x\|^{p}\right\}
$$

is called the duality mapping in $X$ with gauge function $t \rightarrow t^{p-1}$. Since $X$ is uniformly smooth and uniformly convex, $J_{p}$, for each $1<p<\infty$, is single valued, continuous, and strictly monotone. Associated with $J_{p}$, we can introduce the Bregman distance

$$
\Delta_{p}(\bar{x}, x)=\frac{1}{p}\|\bar{x}\|^{p}-\frac{1}{p}\|x\|^{p}-\left\langle J_{p}(x), \bar{x}-x\right\rangle
$$


which is always nonnegative but not necessarily to be a metric.

In Jin (2011c) we generalized the regularizing Levenberg-Marquardt scheme in Hanke (1997) to the Banach space setting. Let $1<p, r<\infty$ and $x_{0} \in D(F)$ be an initial guess. Then, based on the linearized equation (3) around the current iterate $x_{n}$, we consider for each $\alpha>0$ the convex minimization problem

$$
\min _{x \in X}\left\{\frac{1}{r}\left\|y^{\delta}-F\left(x_{n}\right)-F^{\prime}\left(x_{n}\right)\left(x-x_{n}\right)\right\|^{r}+\alpha \Delta_{p}\left(x, x_{n}\right)\right\}
$$

whose unique minimizer is denoted by $x_{n}(\alpha)$. We then define $\alpha_{n}>0$ to be the root of the equation

$$
\left\|y^{\delta}-F\left(x_{n}\right)-F^{\prime}\left(x_{n}\right)\left(x_{n}(\alpha)-x_{n}\right)\right\|=\mu\left\|y^{\delta}-F\left(x_{n}\right)\right\|
$$

for some $0<\mu<1$ and define $x_{n+1}:=x_{n}\left(\alpha_{n}\right)$. The iteration is then terminated by the discrepancy principle (7) which outputs an integer $n_{\delta}$. The convergence of $x_{n_{\delta}}^{\delta}$ to a solution of (1) has been established in Jin (2011c).

Theorem 4. Let $F$ satisfy the tangent cone condition

$$
\left\|F(\bar{x})-F(x)-F^{\prime}(x)(\bar{x}-x)\right\| \leq \eta\|F(\bar{x})-F(x)\|, \quad \bar{x}, x \in B_{\rho}\left(x^{\dagger}\right) \subset D(F)
$$

with $0 \leq \eta<1 / 3$. Let $\eta<\mu<1-2 \eta$ and $\tau>(1+\eta) /(\mu-\eta)$. Then the regularizing LevenbergMarquardt scheme in Banach spaces is well-defined and terminates after $n_{\delta}<\infty$ iteration with $n_{\delta}=$ $O(1+|\log \delta|)$. Moreover $x_{n_{\delta}}^{\delta}$ converges to a solution of (1) as $\delta \rightarrow 0$.

The generalization of the nonlinear Landweber iteration to Banach space setting has been done in Kaltenbacher, Schöpfer and Schuster (2009) and Hein and Kazimierskii (2010). By borrowing the idea from Rieder (1999) we proposed in Jin (2011d) an inexact Newton-Landweber iteration in Banach spaces to accelerate the method by computing more cheap steps in each inner iteration. Let $1 / p+1 / p^{*}=1$, let $J_{p^{*}}^{*}: X^{*} \rightarrow X$ be the duality mapping in $X^{*}$ with gauge function $t \rightarrow t^{p^{*}-1}$, and let $j_{r}: Y \rightarrow Y^{*}$ be a single-valued selection of the duality mapping with gauge function $t \rightarrow t^{r-1}$. Let $x_{n}$ be a current iterate, the method in Jin (2011d) first constructs a sequence $\left\{u_{n, k}\right\} \subset X^{*}$ iteratively by setting $u_{n, 0}=0$ and

$$
u_{n, k+1}=u_{n, k}+\omega_{n, k} T_{n}^{*} j_{r}\left(y-F\left(x_{n}\right)-T_{n}\left(z_{n, k}-x_{n}\right)\right),
$$

where $\omega_{n, k}$ is a positive number and $z_{n, k}=J_{p^{*}}^{*}\left(J_{p}\left(x_{n}\right)+u_{n, k}\right)$. Let $k_{n}$ be the first integer such that

$$
\left\|y-F\left(x_{n}\right)-T_{n}\left(z_{n, k}-x_{n}\right)\right\| \leq \mu\left\|y-F\left(x_{n}\right)\right\|,
$$

where $0<\mu<1$ is a preassigned number, the next iterate is defined to be $x_{n+1}:=z_{n, k_{n}}$. The method is then terminated by the discrepancy principle. Under suitable choice of $\omega_{n, k}$, the method is shown to be strong convergent for the exact data case and weak convergent for the noisy data case.

Finally we want to mention that the iteratively regularized Gauss-Newton method of Bakushinskii has been generalized to Banach space setting in Kaltenbacher, Schöpfer and Schuster (2009) and Kaltenbacher and Hofmann (2010) where the convergence and rates of convergence have been derived under suitable conditions.

\section{REFERENCES}

Bakushinskii, A. B. (1992). The problems of the convergence of the iteratively regularized Gauss-Newton method. Comput. Math. Math. Phys. 32, 1353-1359.

Bauer, F. and T. Hohage (2005). A Lepskij-type stopping rule for regularized Newton methods. Inverse Problems 21, 1975-1991.

Blaschke, B., A. Neubauer and O. Scherzer (1997). On convergence rates for the iteratively regularized Gauss-Newton method IMA J. Numer. Anal. 17, 421-436.

Dembo, R. S., S. C. Eisenstat and T. Steihaug (1982). Inexact Newton methods. SIAM J. Numer. Anal. 19, 400-408. 
Engl, H. W., K. Kunisch and A. Neubauer (1989). Convergence rates for Tikhonov regularization of nonlinear ill-posed problems. Inverse Problems 5, 523-540 (1989)

Hanke, M. (1997). A regularizing Levenberg-Marquardt scheme with applications to inverse groundwater filtration problems. Inverse Problems 13, 79-95.

Hanke, M. (2010). The regularizing Levenberg-Marquardt scheme is of optimal order. J. Integeral Equations and Applications 22 (2), 259-283.

Hanke, M., A. Neubauer and O. Scherzer (1995). A convergence analysis of Landweber iteration of nonlinear ill-posed problems. Numer. Math. 72, 21-37.

Hein, T. and K. S. Kazimierski (2010). Accelerated landweber iteration in Banach spaces. Inverse Problems 26, 055002.

Jin, Q. (2000). On the iteratively regularized Gauss-Newton method for solving nonlinear ill-posed problems. Math. Comp. 69(232), 1603-1623.

Jin, Q. (2011a). A general convergence analysis of some Newton-type methods for nonlinear inverse problems. SIAM J. Numer. Anal. 49, 549-573.

Jin, Q. (2011b). On the order optimality of the regularization via inexact Newton iterations. Numer. Math., to appear.

Jin, Q. (2011c). On the regularizing Levenberg-Marquardt scheme in Banach spaces. preprint 2011.

Jin, Q. (2011d). Inexact Newton-Landweber iteration for solving nonlinear inverse problems in Banach spaces. preprint 2011.

Jin, Q. and Z. Hou (1999). On an a posteriori parameter choice strategy for Tikhonov regularization of nonlinear ill-posed problems. Numer. Math. 83(1), 139-159.

Jin, Q. and U. Tautenhahn (2009). On the discrepancy principle for some Newton type methods for solving nonlinear inverse problems. Numer. Math. 111(4), 509-558.

Jin, Q. and U. Tautenhahn (2011). Inexact Newton regularization methods in Hilbert scales. Numer. Math. 117, 555-579.

Kaltenbacher, B. (1997). Some Newton-type methods for the regularization of nonlinear illposed problems. Inverse Problems 13, 729-753.

Kaltenbacher, B. (1998). A posteriori choice strategies for some Newton type methods for the regularization of nonlinear ill-posed problems. Numer. Math. 79, 501-528.

Kaltenbacher, B. and B. Hofmann (2010). Convergence rates for the iteratively regularized Gauss-Newton method in Banach spaces. Inverse Problems 26, 035007.

Kaltenbacher, B., F. Schöpfer and T. Schuster (2009). Iterative methods for nonlinear ill-posed problems in Banach spaces: convergence and applications to parameter identification problems. Inverse Problems 25, 065003.

Lechleiter, A. and A. Rieder (2010). Towards a general convergence theory for inexact Newton regularizations. Numer. Math. 114(3), 521-548.

Rieder, A. (1999). On the regularization of nonlinear ill-posed problems via inexact Newton iterations. Inverse Problems 15, 309-327.

Rieder, A. (2001). On convergence rates of inexact Newton regularizations. Numer. Math. 88, 347-365.

Scherzer, Q., H. W. Engl and K. Kunisch (1993). Optimal a posteriori parameter choice for Tikhonov regularization for solving nonlinear ill-posed problems. SIAM J. Numer. Anal. 30, 1796-1838.

Tautenhahn, U. and Q. Jin (2003). Tikhonov regularization and a posteriori rules for solving nonlinear ill posed problems. Inverse Problems 19(1), 1-21. 